\title{
Right ventricular multiple myxomas obstructing right ventricular outflow tract
}

Ioannis A. Paraskevaidis, MD, ${ }^{a}$ Konstantinos Triantafilou, MD, ${ }^{\mathrm{b}}$ Dimitrios Karatzas, MD, ${ }^{\mathrm{a}}$ and

Dimitrios Th. Kremastinos, MD, athens, Greece

I

ntracardiac myxoma is the most common tumor of the heart, with an estimated incidence of 0.5 cases per million people per year. ${ }^{1}$ The symptoms vary greatly, depending on the size and location of the tumor. Up to $80 \%$ of myxomas are localized in the left atrium, whereas those emerging from the right side of the heart are rare. To our knowledge, a case of multiple myxomas originating from the right ventricle has never been reported.

\section{Clinical Summary}

A 70-year-old previously healthy woman had a 2-month history of fatigue, worsening exertional dyspnea, frequent dizzy spells, and episodes of loss of consciousness. Three episodes of syncope during exercise were reported. On admission, she had a blood pressure of $130 / 80 \mathrm{~mm} \mathrm{Hg}$ with no signs of right-sided heart failure. On physical examination, a grade 2/6 systolic ejection murmur was audible at the second left parasternal intercostal space. The electrocardiogram showed a normal sinus rhythm of 70 beats/min. The chest $\mathrm{x}$-ray film showed normal results. Transthoracic echocardiography revealed an intracardiac tumor $(9 \times 2 \mathrm{~cm})$ originating in the ventricular-sided basis of the tricuspid valve and producing right ventricular outflow obstruction with a maximal peak gradient of $25 \mathrm{~mm} \mathrm{Hg}$. Transesophageal echocardiography revealed 2 tumors of separate origin in the right ventricle (Figure 1). The first tumor $(2 \times 2 \mathrm{~cm})$ originated in the ventricular-sided basis of the tricuspid valve and prolapsed through it into the right atrium, with no signs of obstruction. The second tumor $(6 \times 3 \mathrm{~cm})$ emerged from the interventricular septum $(2 \mathrm{~cm}$ distally from the first), extended into the right ventricular outflow tract, and prolapsed during systole through the pulmonary valve, causing pulmonary valve obstruction. Coronary arteriography showed no significant lesions of epicardial coronary arteries. Left-sided catheterization was normal. Right-sided catheterization was avoided.
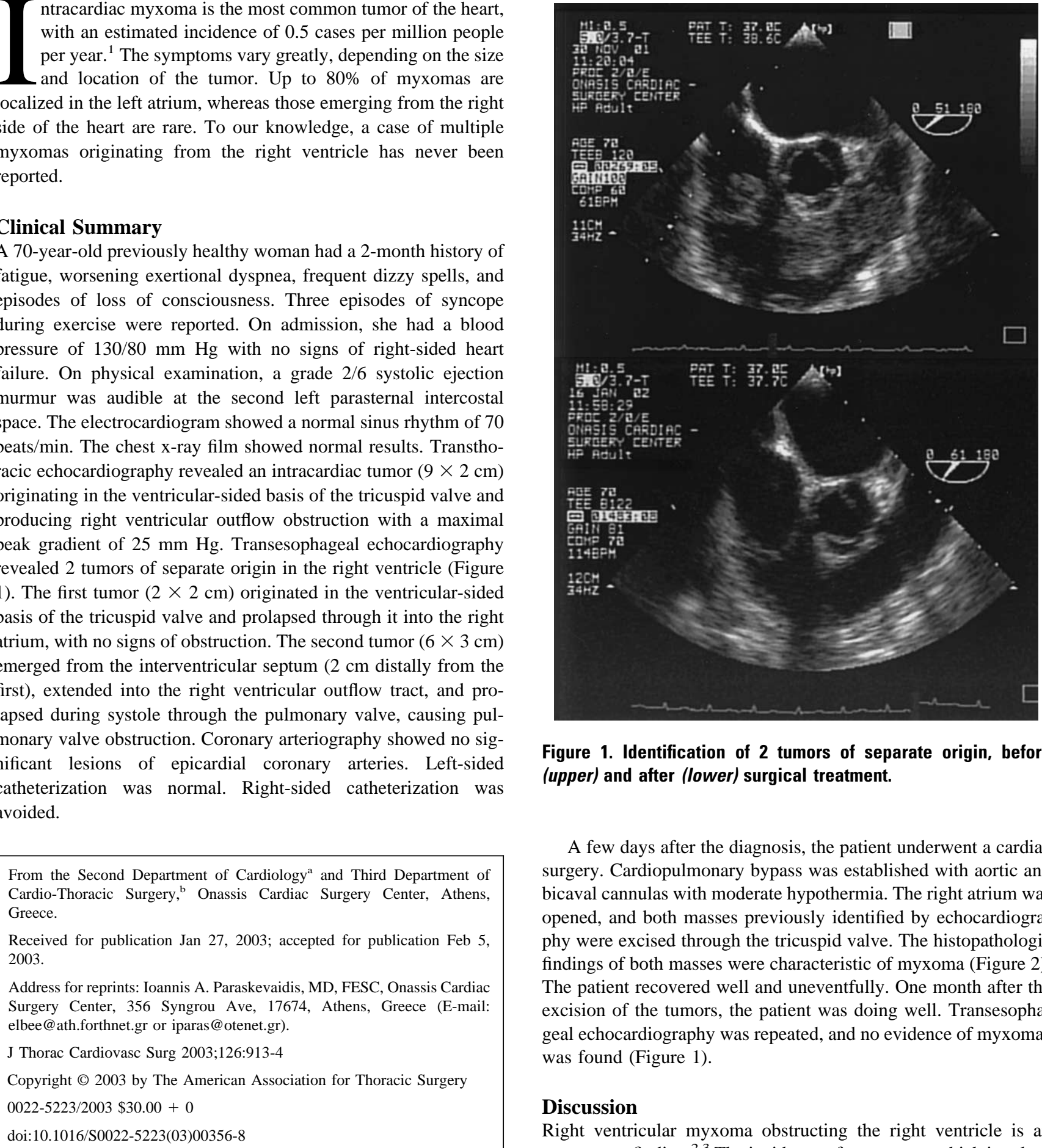

Figure 1. Identification of 2 tumors of separate origin, before (upper) and after (lower) surgical treatment.

A few days after the diagnosis, the patient underwent a cardiac surgery. Cardiopulmonary bypass was established with aortic and bicaval cannulas with moderate hypothermia. The right atrium was opened, and both masses previously identified by echocardiography were excised through the tricuspid valve. The histopathologic findings of both masses were characteristic of myxoma (Figure 2). The patient recovered well and uneventfully. One month after the excision of the tumors, the patient was doing well. Transesophageal echocardiography was repeated, and no evidence of myxomas was found (Figure 1).

\section{Discussion}

Right ventricular myxoma obstructing the right ventricle is an uncommon finding. ${ }^{2,3}$ The incidence of a myxoma, which involves 


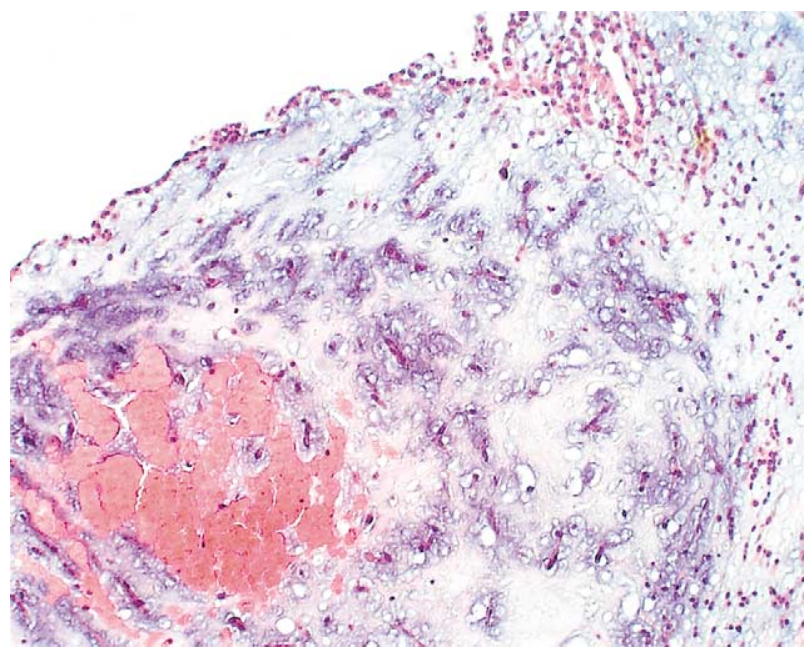

Figure 2. Histopathologic findings of myxomas.

the inflow of the right ventricle and produces right ventricular outflow obstruction, is rare, ${ }^{4}$ and the presence of 2 myxomas originating in the right ventricle involving both right ventricular inflow and outflow tract has never been reported. Symptoms may be variable and are determined by the tumor location and size.
Inflow or outflow obstruction, embolism, syncopal episodes, and arrhythmias are the most common. ${ }^{5}$ After the diagnosis of acute right ventricular obstruction resulting from a cardiac mass, prompt surgical resection is indicated because of imminent embolization and inflow or outflow obstruction, resulting in sudden death. Several surgical techniques have been suggested, but in each case it depends on the site of the tumor.

This case report highlights the fact that right ventricular outflow obstruction associated with episodes of syncope during exercise may be related to a cardiac myxoma, despite the right-sided, very low incidence of this tumor. Transesophageal echocardiography accurately identifies the presence of right ventricular multiple tumors and aids the surgical procedure.

\section{References}

1. MacGowan SW, Sidhu P, Aherne T, et al. Atrial myxoma: national incidence, diagnosis and surgical management. Ir J Med Sci. 1993;162: 223-6.

2. Kern JH, Aguilera FA, Carlson DL, Galantowicz M. Right ventricular myxoma obstructing the right ventricular outflow tract. Circulation. 2000;102:E14-5.

3. Gopal AS, Arora NS, Messineo FC. Right ventricular myxoma. $N$ Engl J Med. 2000;342:295.

4. Krishnamoorthy KM, Desai NB. Myxoma producing right-sided inflow and outflow obstruction. Int J Cardiol. 2001;79:325-6.

5. Silvermann NA. Primary cardiac tumors. Ann Surg. 1980;191:127-38.

\title{
Origin of atrial fibrillation from the pulmonary veins in a mitral patient
}

\author{
Joao 0. Melo, MD, PhD, Stefano Benussi, MD, PhD, Walter Tortoriello, MD, Vicenzo Santinelli, MD, Simone Calvi, MD, \\ Simona Nascimbene, MD, Carlo Pappone, MD, and Ottavio Alfieri, MD, Milan, Italy
}

$\mathrm{I}$ $\mathrm{n}$ mitral patients the origin and mechanisms of atrial fibrillation are largely unknown. Even though several theories have been postulated for its origin, there is scarce evidence to support them. Surgical procedures that electrically isolate the pulmonary veins from the left atrial chamber are associated with a very high success rate. We report a case of a mitral patient with permanent atrial fibrillation who underwent mitral valve replacement and extended bilateral isolation of the pulmonary veins using intraoperative radiofrequency ablation. After surgery,

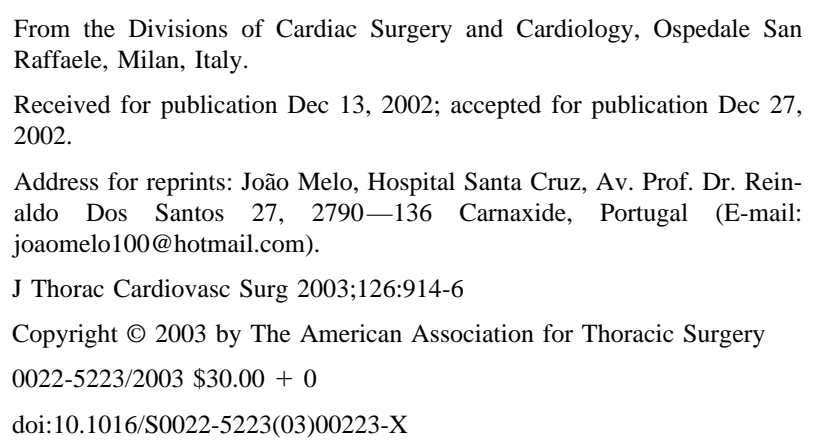

bidirectional electrical block from the right pulmonary veins was documented.

Postoperative epicardial recordings and Holter monitoring showed paroxysmal tachycardia limited to the atrial cuff of the right pulmonary veins while the patient remained in stable sinus rhythm.

\section{Clinical Summary}

This 56-year-old female patient had severe mitral valve stenosis with moderate regurgitation, tricuspid regurgitation, and permanent atrial fibrillation of 10 months' duration. She was in New York Heart Association class functional II, with a peak systolic pulmonary artery pressure of $50 \mathrm{~mm} \mathrm{Hg}$ and moderate tricuspid regurgitation.

Under cardiopulmonary bypass and moderate hypothermia, the mitral valve was replaced, preserving the posterior leaflet, with a St Jude Medical 27 prosthesis (St Jude Medical, Inc, St Paul, Minn), and the tricuspid valve was repaired with a Kay annuloplasty.

Myocardial protection was achieved with antegrade and retrograde blood cardioplegia. Before the valve procedure, extended bilateral isolation of pulmonary veins as previously described by 